\title{
Microscopic Modeling of Nonlinear Transport in Ballistic Nanodevices
}

\author{
Javier Mateos, B. G. Vasallo, Daniel Pardo, Tomás González, Jean-Sébastien Galloo, Sylvain Bollaert, \\ Yannick Roelens, and Alain Cappy
}

\begin{abstract}
By using a semi-classical two-dimensional (2-D) Monte Carlo simulation, simple ballistic devices based on AlInAs/InGaAs channels are analyzed. Our simulations qualitatively reproduce the experimental results in $\mathrm{T}$ - and $\mathrm{Y}$-branch junctions as well as in a ballistic rectifier appearing as a result of electron ballistic transport. We show that a quantum description of electron transport is not essential for the physical explanation of these results since phase coherence plays no significant role. On the contrary, its origin can be purely classical: the presence of classical electron transport and space charge inside the structures.
\end{abstract}

Index Terms-Ballistic transport, Monte Carlo simulation, terahertz devices.

\section{INTRODUCTION}

$\mathbf{O}$ $\mathrm{NE}$ of the possible approaches for overcoming the limits of traditional scaling when reaching the nanometer range (which has been the main engine of the progress of the semiconductor industry) is the use of devices exploiting the ballistic transport of electrons. Ballistic devices fabricated using the GaAs/AlGaAs heterojunction operating at low temperature have been demonstrated [1], [2]. However, recent works have achieved an important improvement using InGaAs channels with high In content [3]-[6], room temperature operation is possible since the mean-free-path of electrons is still larger than $100 \mathrm{~nm}$, which is a feature size in the reach of current lithographic techniques. The small size of these ballistic devices and the high velocity of electrons inside reduce significantly their transit time, and as a result, the fabrication of devices for data processing at ultra-high bit rate can be envisaged [4], [5]. Moreover, InGaAs-based ballistic devices offer the advantage of being compatible with modern HEMT technology; indeed, AlInAs/InGaAs HEMTs nowadays operate in the millimeter and submillimeter wave frequency range [7]. Thus, the integration of ballistic devices with HEMTs in order to benefit from their complementary advantages and reach the terahertz regime appears feasible in the near future.

Manuscript received October 22, 2002; revised May 23, 2003. This work was supported in part by the European Commission through the NANOTERA under Project IST-2001-32517, by the Dirección General de Investigación (Ministerio de Ciencia y Tecnología) and FEDER under Project TIC2001-1754, and by the Consejería de Cultura de la Junta de Castilla y León under Project SA057/02. The review of this paper was arranged by Editor H. Sakaki.

J. Mateos, B. G. Vasallo, D. Pardo, and T. González are with the Universidad de Salamanca, Salamanca, Spain (e-mail: javierm@usal.es).

J.-S Galloo, S. Bollaert, Y. Roelens, and A. Cappy are with the Institut d'Electronique de Microélectronique et de Nanotechnologie U.M.R. C.N.R.S., Département Hyperfréquences et Semiconducteurs, Villeneuve D’Ascq Cédex, France.

Digital Object Identifier 10.1109/TED.2003.815858
The first step in the design of ballistic structures is the determination of their optimal geometry. At this level, simulation tools constitute a valuable alternative to the expensive and timeconsuming test-and-error procedure. Some theoretical descriptions of the operation of ballistic devices have been proposed [8]-[10], [19], always starting from a coherent transport description based on the Landauer-Buttiker formalism [11], [12]. In this paper, we present a microscopic analysis, performed by means of Monte Carlo (MC) simulations, of the transport properties of several structures based on AlInAs/InGaAs ballistic channels specially designed to be applied in electronic devices for terahertz data processing. MC simulations provide an insight of the processes taking place inside the devices, thus allowing us to relate the macroscopic results of the experiments with the microscopic behavior of electrons. Our model, which is based on a semiclassical transport description, is able to qualitatively reproduce the main features of the ballistic effects measured in basic devices like T-branch (TBJs) [6] and Y-branch (YBJs) junctions [1], [3] and ballistic rectifiers [2], [4], thus demonstrating that coherent transport plays no significant role on the main characteristics of these devices.

In Section II, the details about the MC model and the simulated structures will be given. In Section III, the validity of our approach will be checked by comparison of the simulations with measurements performed in real AlInAs/GaInAs channels, with the layer structure typically used in the fabrication of HEMTs. Then, in Section IV, simulations of ballistic TBJs, YBJs, and ballistic rectifiers will be presented, qualitatively reproducing the main experimental findings shown in the literature [1]-[4], [6].

\section{Monte CARLo Model}

We make use of a semiclassical ensemble MC simulator selfconsistently coupled with a 2-D Poisson solver. The transport model locally takes into account the effect of degeneracy and electron heating by using the rejection technique and the selfconsistent calculation of the local electronic temperature and Fermi level [13]. The surface charges appearing at the boundaries of the semiconductors in contact with dielectrics are also considered in the model [14]. The validity of this approach has been checked in previous works by means of the comparison with experimental results of static characteristics, small signal behavior, and noise performance of a $0.1-\mu \mathrm{m}$ gate AlInAs/InGaAs lattice matched HEMT (InP based) [14], [15]. Since contact injection is a critical point when dealing with ballistic transport, the velocity distribution and time statistics of injected carriers will be accurately modeled [16], [20]. 


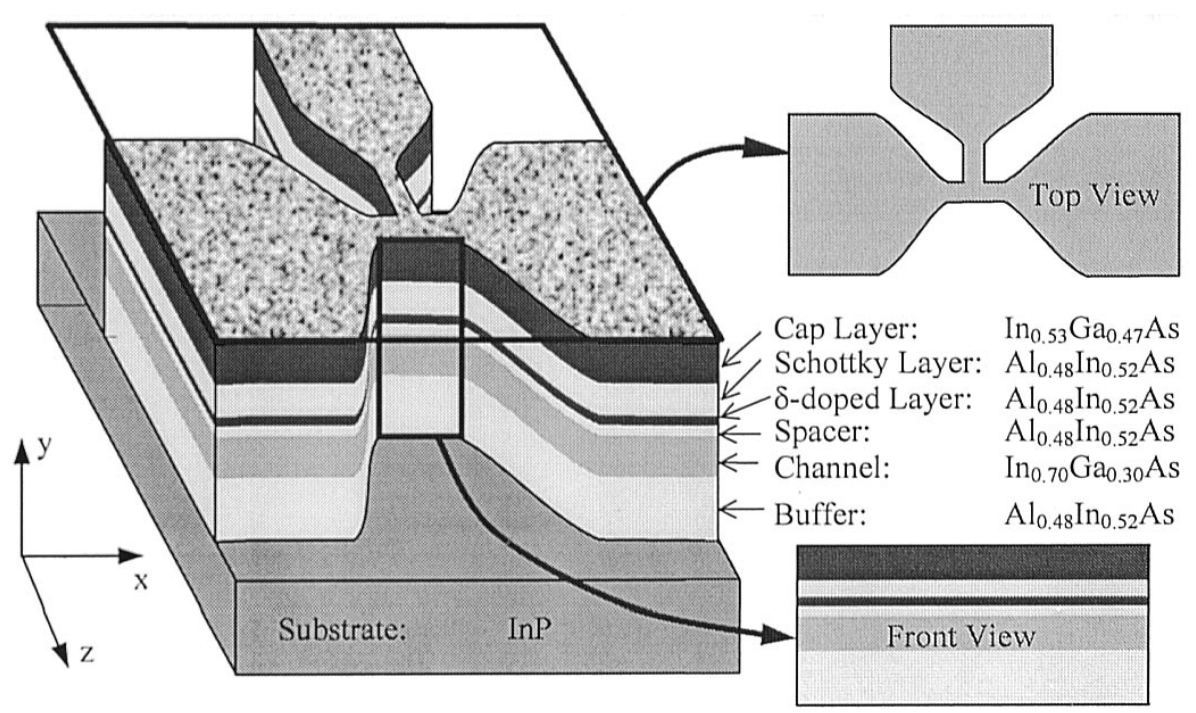

Fig. 1. Three-dimensional (3-D) geometry and layer structure of the ballistic channels and scheme of the 2-D front-view (FV) and top view (TV) Monte Carlo simulations.

The main features of the ballistic channels that will be simulated are shown in Fig. 1, where the geometry is usually defined in the fabrication process by a typical mesa etching. The real layer structure fabricated by molecular beam epitaxy (MBE) consists of an InP substrate, a 200-nm $\mathrm{Al}_{0.48} \mathrm{In}_{0.52} \mathrm{As}$ buffer followed by a 15 -nm-thick $\operatorname{In}_{0.7} \mathrm{Ga}_{0.3} \mathrm{As}$ strained channel, three layers of $\mathrm{Al}_{0.48} \mathrm{In}_{0.52} \mathrm{As}$ (a 5-nm spacer, a $\delta$-doped layer, and a 10 -nm Schottky layer), and finally, a 15 -nm-thick $\operatorname{In}_{0.53} \mathrm{Ga}_{0.47}$ As cap layer $\left(N_{D}=6 \times 10^{18} \mathrm{~cm}^{-3}\right)$. Layers with two different $\delta$-dopings have been fabricated (4 and $5 \times 10^{12} \mathrm{~cm}^{-2}$ ), and they will be modeled as a 4-nm layer doped at $10^{19} \mathrm{~cm}^{-3}$ for the $\delta=4 \times 10^{12} \mathrm{~cm}^{-2}$ and $1.25 \times 10^{19} \mathrm{~cm}^{-3}$ for the $\delta=5 \times 10^{12} \mathrm{~cm}^{-2}$ (moreover, a structure with $\delta=6 \times 10^{12} \mathrm{~cm}^{-2}$ will be also simulated using $N_{D}=1.5 \times 10^{19} \mathrm{~cm}^{-3}$ ). To account for the thickness of the $\delta$-doped layer while keeping the overall size, the dimension of the spacer and Schottky layers are set to 3 and $8 \mathrm{~nm}$, respectively.

For the correct modeling of these devices, a 3-D simulation would be necessary in order to take into account the effect of the lateral surface charges and the real geometry of the structures. However, for the moment, only a 2-D MC model has been developed, and some simplifications and assumptions must be made. Indeed, two different types of 2-D simulations will be performed: front-view (FV) and top-view (TV). Within the FV simulations, the layer structure will be taken into account, but the device in the $z$ dimension is considered to be homogeneous. This kind of simulations will be useful for simple structures, like homogeneous channels, and will provide the concentration of carriers in each layer. On the other hand, to account for the top geometry of more complicated devices (such as TBJs, YBJs, or ballistic diodes), TV simulations will be carried out. They are performed in the $x y$ plane; therefore, the real layer structure is not included, and only the InGaAs channel will be simulated. In order to account for the fixed positive charges of the whole layer structure, a net doping is assigned to the channel in TV simulations, but impurity scattering is switched off. In this way, the electron transport through the undoped channel is well repro- duced since this is a "virtual" doping associated with the charges of the cap and $\delta$-doped layers. On the other hand, a negative surface charge density is assigned to the semiconductor-air interfaces to account for the influence of the surface states originated by the etching processes. Therefore, to ensure the accuracy of this TV approximation, the values of two important parameters must be carefully chosen: the background doping in the channel $N_{D b}$ and the lateral surface charge density $\sigma$.

\section{BALLISTIC CHANNELS}

Initially, the validity of the simulation tools is checked by comparison of the numerical results with experimental Hall-effect measurements of carrier concentration and mobility of two fabricated layers $\left(\delta=4 \times 10^{12} \mathrm{~cm}^{-2}\right.$ and $\left.\delta=5 \times 10^{12} \mathrm{~cm}^{-2}\right)$. To improve the ballistic character of electron motion, impurity scattering will be diminished by allowing transport only through the InGaAs channel. For this sake, recessed channels (the cap layer is removed) will be used. By adjusting the surface charge at the cap layer to a value of $\sigma_{\text {cap }} / \mathrm{q}=4.7 \times 10^{12} \mathrm{~cm}^{-2}$, and at the bottom of the recess (free AlInAs interface) to $\sigma_{\text {rec }} / \mathrm{q}=$ $2.8 \times 10^{12} \mathrm{~cm}^{-2}$, we have obtained a good agreement with the measured values of Hall density in both recessed and nonrecessed layers. However, we have appreciated that the channel mobility obtained by MC simulations overestimates the measured values (around $10000 \mathrm{~cm}^{2} / \mathrm{Vs}$ ). We have checked that this discrepancy is due to remote impurity scattering (not included in the MC model) since the experimental mobility of the channel is improved when a thicker spacer layer is growth, reaching a better agreement with the MC values (around 14000 $\mathrm{cm}^{2} / \mathrm{Vs}$ ).

We have performed the FV simulation of two different channels (considering the whole layer structure) with lengths $L=$ $1000 \mathrm{~nm}$ and $L=100 \mathrm{~nm}$ between two ohmic contacts. The diffusive or ballistic character of transport depending on $L$ can be monitored, from the point of view of the Monte Carlo simulation, by means of the number of scattering processes the electrons overcome while crossing the sample. In practice, trans- 


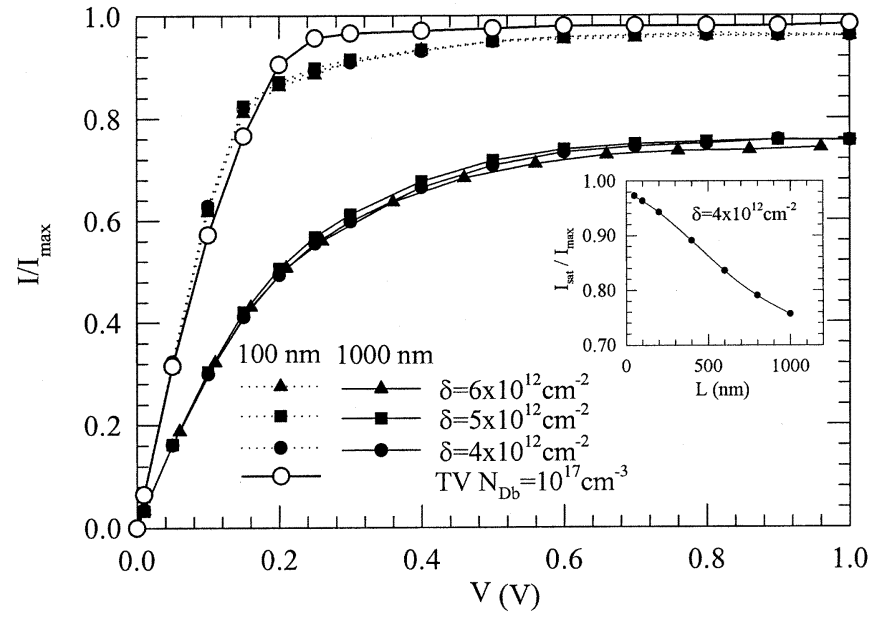

Fig. 2. Normalized current versus applied voltage for nonrecessed channels with lengths of $100 \mathrm{~nm}$ (dotted lines) and $1000 \mathrm{~nm}$ (solid lines) and different $\delta$ dopings. The values obtained from TV simulations with $N_{D b}=10^{17} \mathrm{~cm}^{-3}$ and $L=100 \mathrm{~nm}$ are also shown. The inset shows the normalized saturation current as a function of the channel length for $\delta=4 \times 10^{12} \mathrm{~cm}^{-2}$.

port will never be completely ballistic since, even for very short $L$, electrons always overcome a few scattering mechanisms. Some other features can give us information about how ballistic the transport inside the channels is, ranging from diffusive (for the channel with $L=1000 \mathrm{~nm}$ ) to quasiballistic ( $L=$ $100 \mathrm{~nm}$ ). One of these indicators is the ratio between the saturation current $I_{s}$ and the total current injected by the contacts $I_{\max }$, which is the maximum current that may flow through the channels [16], [20]. In the case of completely ballistic transport, $I_{s}=I_{\max }$ since every electron injected by the cathode arrives at the anode (for high enough biasing). When scattering mechanisms appear, some carriers return to the anode, making the ratio $I_{s} / I_{\max }$ lower than unity. In Fig. $2, I / I_{\max }$ is represented as a function of the applied voltage, showing that $I_{s} / I_{\max }$ increases when reducing $L$ due to the lower amount of scattering mechanisms, which leads to a more pronounced velocity overshoot originated by the enhanced ballistic transport. The value of $I_{s} / I_{\max }$ as a function of $L$ is plotted in the inset of Fig. 2, showing that for $L<200 \mathrm{~nm}, I_{s} / I_{\max }$ exceeds $95 \%$, and electron transport can be considered to be quasiballistic. We have to note that the results plotted in Fig. 2 correspond to nonrecessed channels (including the cap layer). In the case of recessed channels, impurity scattering in the cap layer is prevented, and the ratio $I / I_{\max }$ is increased but only for the long channels. The values of $I / I_{\max }$ for the 100 -nm nonrecessed and recessed channels overlap since the mean free path associated with ionized impurity scattering is longer than the length of the structures. Therefore, the recessed technology will be useful for improving the ballistic transport only when the length of the channels approaches the ballistic/diffusive limit (around $200 \mathrm{~nm}$ ).

The simulation of the ballistic channels can also be made within the TV approach, considering a background doping $N_{D b}$ with no impurity scattering. In Fig. 2, the inductance-voltage $(I-V)$ curve obtained with this model is compared with the correct FV simulations of the 100-nm channels. In this case, to compare with the FV results, no lateral surface charge is considered since this charge can not be accounted for in FV simu-

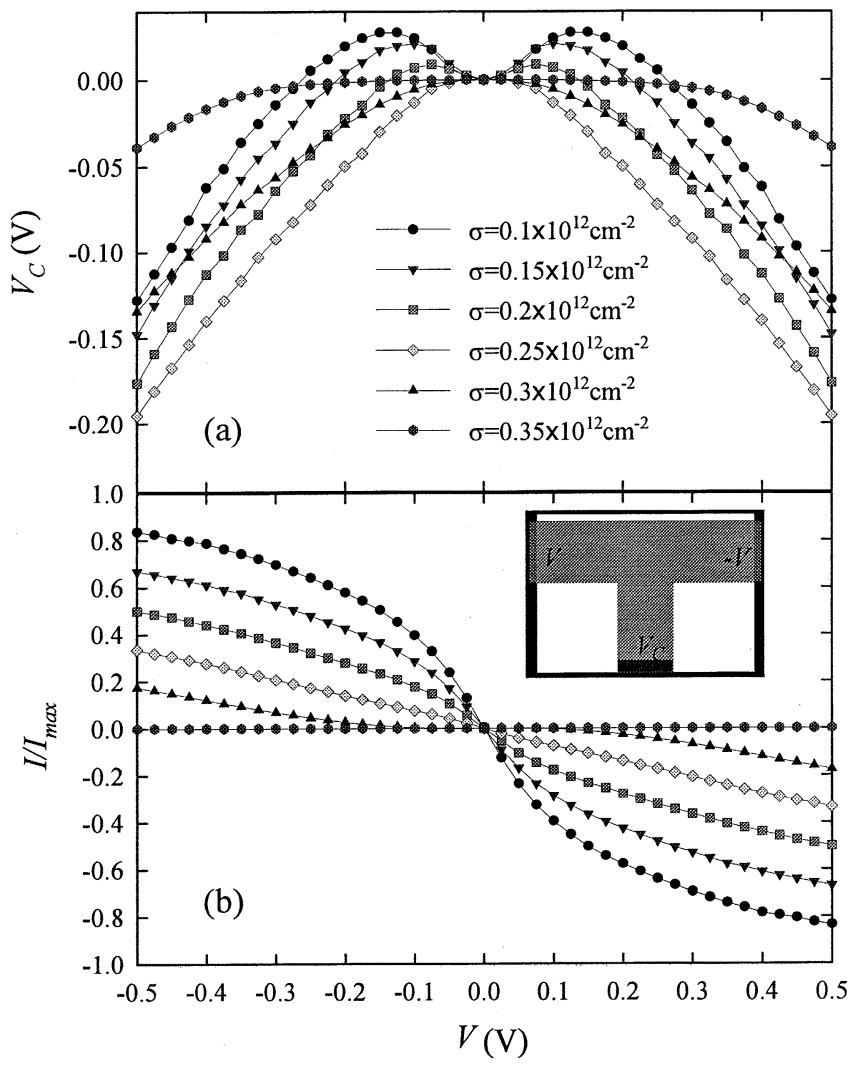

Fig. 3. (a) Electric potential at the bottom of the central branch (open circuited) of the TBJ and (b) normalized horizontal current when biasing the left and right contacts in push-pull fashion $\left(V=V_{L}=-V_{R}\right)$ for different values of the lateral surface charge. The inset shows the geometry of the TBJ with 50-nm-wide and 75-nm-long branches.

lations. It can be appreciated that setting the background doping to a value $N_{D b}=10^{17} \mathrm{~cm}^{-3}$ and considering injecting contacts with $N_{c}=4 \times 10^{17} \mathrm{~cm}^{-3}$ [16], [20], TV simulations satisfactorily reproduce the behavior of the ballistic channels (both recessed and nonrecessed). These values will therefore be used in all further TV simulations.

\section{BASIC DEVICES}

\section{A. T-Branch Junctions}

As a first example of devices that benefit from ballistic transport, we will analyze the main features of a TBJ. In [6], the experimental measurements of the negative potential generated at the bottom of the central branch (open circuited, $I_{c}=0$ ) of a TBJ $\left(V_{C}\right)$ when the left and right ones are biased in push-pull fashion $\left(V=V_{L}=-V_{R}\right)$ are presented. As stated in [10] and [19], this property of the three terminal ballistic junctions (both TBJs and YBJs) can be very useful from a practical point of view since it can be exploited to perform logical operations [17], second harmonic generation [5], [18] or rectification [2], [4].

In Fig. 3(a), we show the values of $V_{C}$ calculated with TV-MC simulations of the TBJ sketched in the inset considering different values for the lateral surface charge density $\sigma$. The value of $V_{C}$ at equilibrium has been subtracted from the results to account for the difference of electrochemical potentials. The simulations do qualitatively reproduce the experimental 


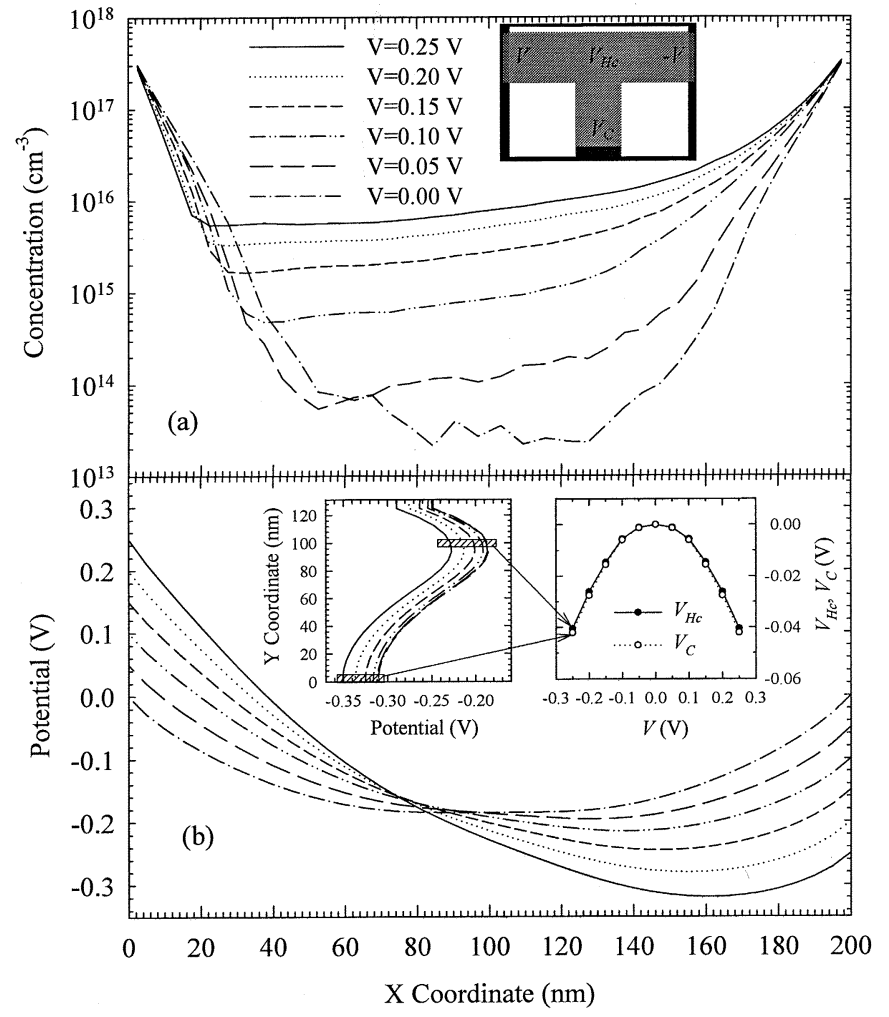

Fig. 4. (a) Electron concentration and (b) electric potential profiles along the middle of the horizontal branch of the TBJ of Fig. 3 with $\sigma=0.3 \times 10^{12} \mathrm{~cm}^{-3}$ for different bias conditions $\left(V=V_{L}=-V_{R}\right.$ ). The insets of (b) show the vertical potential profile in the middle of the central branch for several biasings, and the values of the potential at the bottom of this branch $V_{C}$ and at the center of the junction $V_{H c}$ as a function of $V$.

results, thus also supporting the validity of our TV-MC model. As seen in Fig. 3, the values of $V_{C}$ depend on the amount of surface charge considered at the semiconductor-air interfaces since it controls the intensity of space charge effects [the amplitude of the potential minimum observed in Fig. 4(b)]. The effect of the surface charge is similar to that observed in [6] by changing the potential of a top gate contact, both affecting the Fermi level pinning and the electron concentration inside the TBJ. The correct values of the background doping and surface charge density to be used in the TV-MC simulation will have to be further adjusted, taking as a base the experimental measurements of channels with different length and width that we plan to fabricate. Nevertheless, as pointed out in [4], the lateral depletion length of InGaAs channels $L_{d}$ is about 10-30 nm, which corresponds to surface charge densities $\sigma$ of $0.1-0.3 \times 10^{12} \mathrm{~cm}^{-3}$ (using $N_{D b}=10^{17} \mathrm{~cm}^{-3}$ ), which is in reasonable agreement with the range of values used in our simulations.

The negative values of $V_{C}$ are related to space-charge effects originated by the joint action of the surface charge at the semiconductor-air interfaces, the background positive fixed charge $N_{D b}$, and the inhomogeneous charge distribution associated with the ballistic motion of carriers injected at the contacts. The surface charge lowers the electric potential when moving away from the contacts, provoking the progressive depletion of the channel, thus leading to the typical minimum of potential and concentration (see Fig. 4) characteristic of space-charge limited

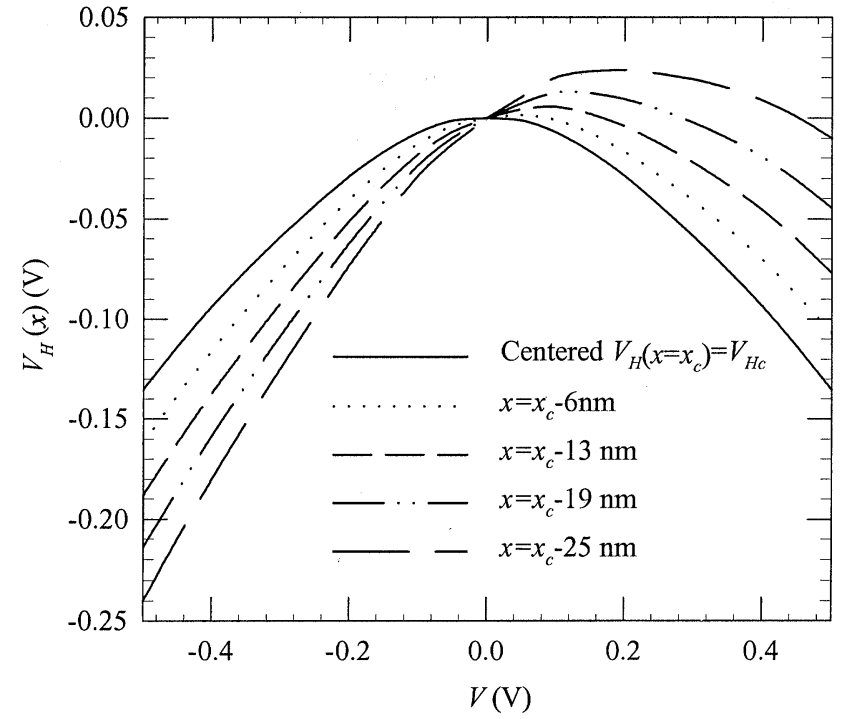

Fig. 5. Electric potential at different positions of the horizontal channel of the TBJ of Fig. 3 as a function of the biasing $\left(V=V_{L}=-V_{R}\right)$.

ballistic conditions [16], [20]. At equilibrium, this minimum is in the middle of the structure. When the TBJ is biased, the carrier number inside the device increases since more and more electrons are able to surmount the potential barrier (i.e., more modes are opened under the Landauer-Buttiker description [8]-[10], [19]), leading to the nonlinear $I-V$ characteristic shown in Fig. 3(b). Moreover, the concentration exhibits an asymmetric shape (higher near the negative electrode due to the electron ballistic motion) so that a shift of the potential minimum toward the negative electrode takes place. As a consequence, the potential at the center of the horizontal channel $V_{H}\left(x_{c}\right)=V_{H c}$ is always lower than the equilibrium value and decreases with larger $V$. The inset of Fig. 4(b) shows the vertical profile of the electric potential in the middle of the central branch. It can be observed that the variations of $V_{H c}$ versus $V$ propagate down to the bottom of the vertical branch, thus originating the characteristic bell-shaped values of $V_{C}$. Therefore, the vertical branch acts just like a voltage probe connected to the horizontal channel, detecting the potential variations at the junction. This happens because the penetration of carriers in the central branch is just the consequence of the nonzero vertical velocity component of the carriers flowing within the horizontal channel, thus being almost independent of $V$. In the case that the vertical branch is slightly shifted from the center of the horizontal channel, the potential at the junction $V_{H}(x)$, and therefore $V_{C}$, are no longer symmetric, taking positive values for some values of $V$ (Fig. 5), as already predicted in [10] and [19] for asymmetric junctions.

In Fig. 3, we can also observe that the negative values of the $V_{C}$ versus $V$ curve reach a maximum for an intermediate value of $\sigma$, just when the width of the channel coincides with the lateral depletion induced by the surface charge (for $\sigma=$ $0.25 \times 10^{12} \mathrm{~cm}^{-3}, L_{d}=25 \mathrm{~nm}$, and the theoretical effective width of the channel $W_{\text {eff }}=W-2_{L d}$ becomes 0 ). In Fig. 6(a), it is shown that under these conditions, and for low values of $V, V_{C}$ follows the predicted quadratic dependence on $V$ [10], [19], but it becomes linear for high $V$. In Fig. 6(b), the 


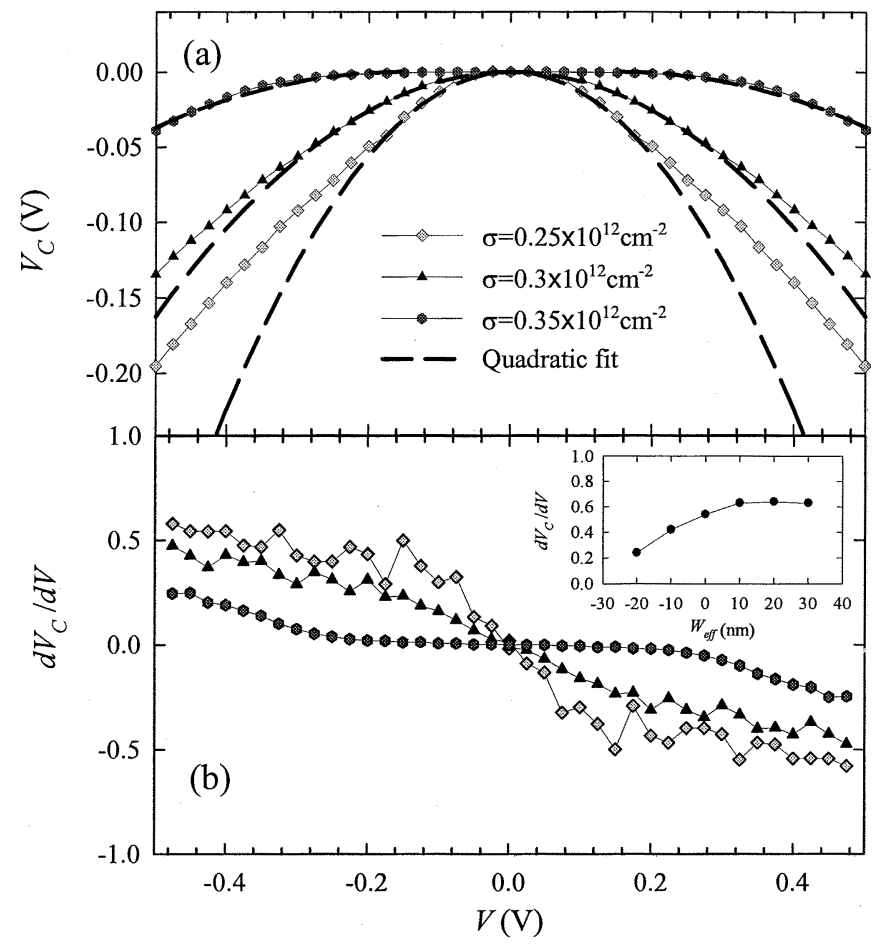

Fig. 6. (a) Quadratic fit of $V_{C}(\mathrm{~V})$ and (b) $d V_{C} / d V$ for different values of $\sigma$. The inset shows the value of $d V_{C} / d V$ as a function of $W_{\text {eff }}$ for $V=0.5 \mathrm{~V}$.

slope of $V_{C}(V)$ is plotted, first showing a linear increase (corresponding to the parabolic increase of $V_{C}$ ) and then saturating for high $V$ (in the linear portion of the curve). The value of $d V_{C} / d V$ for a constant $V$ (inset of Fig. 6) increases with $W_{\text {eff }}$ for $W_{\text {eff }}<0$ (strong space-charge effects) and reaches a saturation value when $W_{\text {eff }}>0$.

If $\sigma$ is increased, $W_{\text {eff }}$ becomes lower than 0 (for example, $\sigma=0.35 \times 10^{12} \mathrm{~cm}^{-3}$ leads to $W_{\text {eff }}=-20 \mathrm{~nm}$ ), meaning that the potential barrier is too high to be surmounted by any carrier at equilibrium. The asymmetry in the electron concentration for low values of $V$ is very small, and $V_{C}$ approaches $\left(V_{L}+V_{R}\right) / 2=0$. When strongly biasing the TBJ, some carriers can flow through the device, and thus, the negative values of $V_{C}$ emerge only for high $V$ (with the characteristic parabolic shape). On the other hand, if $\sigma$ is reduced ( $W_{\text {eff }}>0$ ), some alteration on the bell shape of $V_{C}$ is observed for low $V$, where $V_{C}$ even takes positive values due to the perturbation induced by the central branch on the minimum of the horizontal potential profile. This perturbation could be avoided by reducing the width of the central branch.

It is important to remark that all these results are a consequence of purely classical effects related to the ballistic motion of electrons and Coulomb interaction, with no essential role played by phase coherence. Therefore, a quantum transport description, as adopted in [10] and [19], is not strictly necessary for the modeling of these kinds of devices.

\section{B. Y-Branch Junctions}

Let us now study a slightly different ballistic device: the YBJ (a TBJ can be considered a particular case of a YBJ with an opening angle between left and right branches of $\left.180^{\circ}\right)$. As reported in [3], if the potential at the central branch $\left(V_{C}\right.$, with
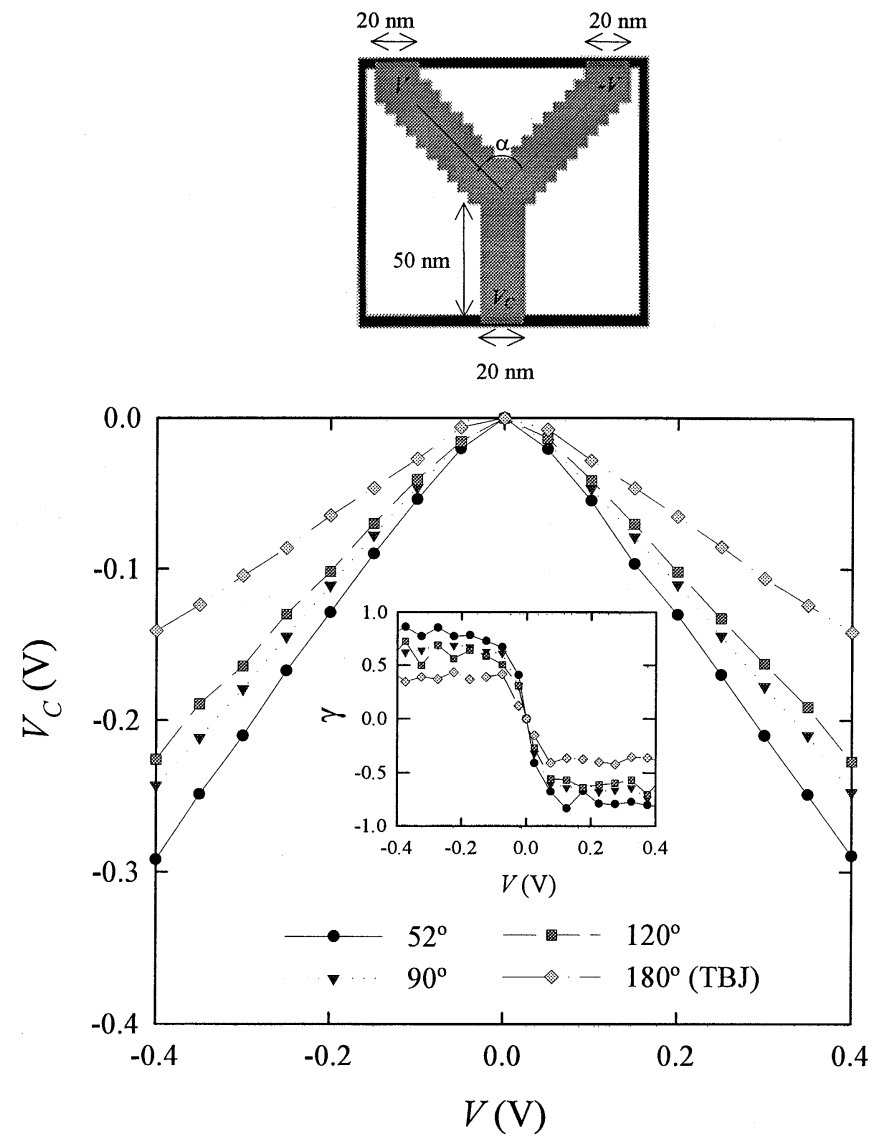

Fig. 7. $V_{C}$ as a function of the biasing $\left(V=V_{L}=-V_{R}\right)$ in YBJs with 20-nm-wide and 50-nm-long branches with $\sigma=0.1 \times 10^{12} \mathrm{~cm}^{-2}\left(W_{\text {eff }}=\right.$ 0 ) and different opening angles. The inset shows the value of the switching parameter $\gamma=d V_{C} / d V$ as a function of $V$. The geometry of the YBJ with $\alpha=90^{\circ}$ is sketched at the top.

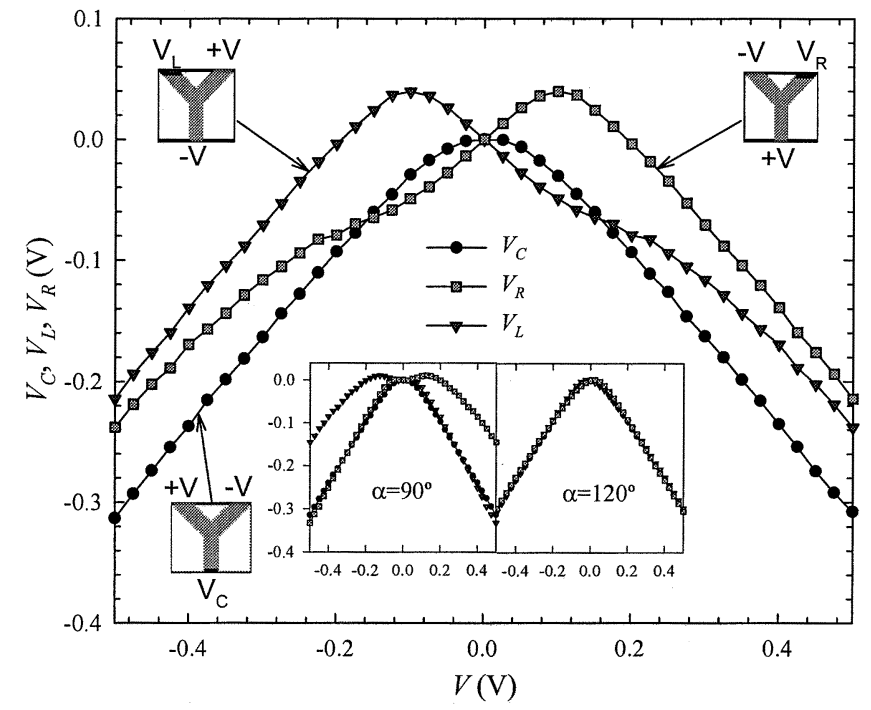

Fig. 8. $V_{C}, V_{L}$, and $V_{R}$ as a function of $V$ when biasing with $V=V_{L}=$ $-V_{R}, V=V_{R}=-V_{C}$, and $V=V_{C}=-V_{L}$, respectively, a YBJ with 50-nm-wide branches, $\alpha=90^{\circ}$, and $\sigma=0.25 \times 10^{12} \mathrm{~cm}^{-2}\left(W_{\text {eff }}=0\right)$. The length of the central branch is $125 \mathrm{~nm}$, whereas that of the left and right ones is $75 \mathrm{~nm}$. The two insets show the results for two symmetric (three $75 \mathrm{~nm}$ long branches) YBJ, with $\alpha=90^{\circ}$ and $\alpha=120^{\circ}$, respectively.

$\left.I_{c}=0\right)$ of a YBJ is measured when biasing the left and right branches with $V=V_{L}=-V_{R}$, it shows the same qualitative 


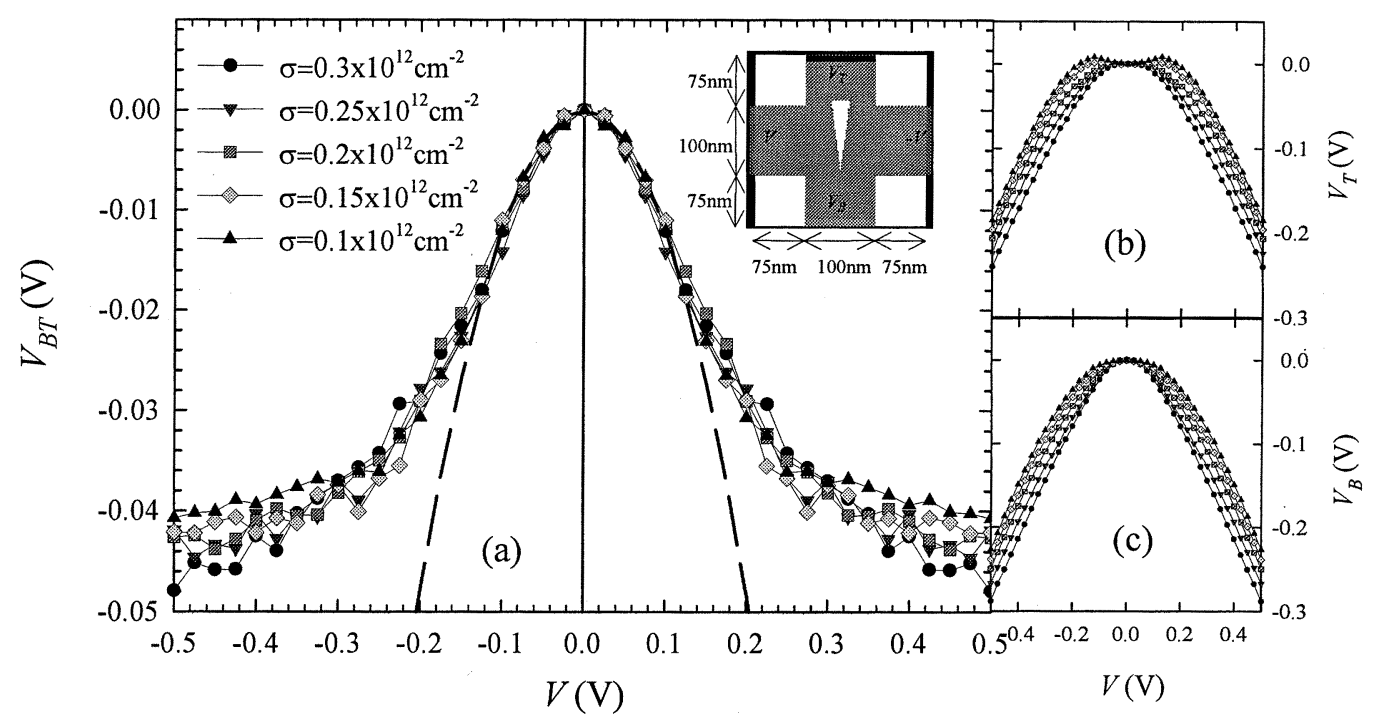

Fig. 9. (a) $V_{B T}$, (b) $V_{T}$, and (c) $V_{B}$ as a function of $V$ when biasing with $V=V_{L}=-V_{R}$. The ballistic rectifier with the geometry is shown in the inset.

behavior as that discussed before for the TBJ. However, one important difference exists: As $V$ is enlarged, the electrons penetrate more and more into the central branch (stem), pushed by the increasing electric field (and not only because of the vertical thermal velocity component as in the TBJ), thus making $V_{C}$ decrease further. As a consequence, the electron concentration in the stem grows significantly when increasing $V$, enhancing the curvature of the $V_{C}$ versus $V$ curve. The bell shape of $V_{C}$ is therefore the addition of two effects: i) the horizontal behavior of the potential (as shown for the TBJ) and ii) the vertical penetration of carriers into the central branch. The first is just an electrostatic effect, and as predicted in [10] and [19], it depends only on the conductance of each of the three branches, being independent of the angle between the left and right branches. On the contrary, the second depends on the amount of carriers injected into the stem and, therefore, on the angle between left and right branches $\alpha$ having a maximum effect for $0^{\circ}$ and minimum for $180^{\circ}$ (TBJ case). In Fig. 7, the results of the MC simulations for both $V_{C}$ and the so-called switching parameter $\gamma=d V_{C} / d V$ are plotted [3] for YBJs with different opening angles. When increasing $V, V_{C}$ passes from a parabolic to a linear behavior, in the same way as observed before for the TBJ. Consequently, $\gamma$ increases linearly for low $V$ and then saturates for high $V$, showing a remarkable agreement with the experimental results of [3]. It can be also appreciated that the decrease of $\alpha$ (from the $180^{\circ}$ value of the TBJ) enhances the negative values of $V_{C}$ (making the saturation value of $\gamma$ increase from 0.4 for the TBJ to 0.8 for the YBJ with $\alpha=52^{\circ}$ ), due to the stronger injection of carriers into the central branch.

Our model can also explain the asymmetric behavior of the potential measured in the left (right) branch of a YBJ when biasing right and central (central and left) branches, as shown in Fig. 8 for a YBJ with $\alpha=90^{\circ}$. The asymmetry of these curves is not only due to the more or less straight path of the electrons (as it was claimed in [3]) that affects the penetration of carriers into the left (right) branch but also to the different length of the left and right branches as compared with the central one (in this case, it is $50 \mathrm{~nm}$ longer). The asymmetry associated with the different length of the branches has an effect similar to that observed in Fig. 5 for the TBJ, leading even to positive values of the potential in the open-circuited lead. Moreover, as shown in Fig. 3, positive voltages can also appear due to a large $W_{\text {eff }}$. On the other hand, the asymmetry of the electron injection into the open-circuited branch affects mainly the slope of the measured voltage. This can be easily explained with the help of the two insets of Fig. 8, showing the value of the potentials in the open-circuited lead both for $\alpha=90^{\circ}$ and $\alpha=120^{\circ}$ when the lengths of the three branches are identical. For the YBJ with $\alpha=90^{\circ}$, the behavior of $V_{L}$ and $V_{R}$ is different for positive and negative values of $V$. Let us explain the dependence of $V_{L}$ on $V$. When applying a negative voltage to $V_{R}$ and positive to $V_{C}\left(V_{R}=-V_{C}=V<0\right), V_{L}$ behaves similarly to the case of the TBJ since few electrons (fewer than in a TBJ because of the orientation of the biased branches) are injected into the left branch, leading to small positive values of $V_{L}$ for low $V$ (even if $\left.W_{\text {eff }}=0\right)$. When biasing inversely $\left(V_{R}=-V_{C}=V>0\right)$, $V_{L}$ decreases faster with $V$ since the biasing favors the injection into the left branch. Indeed, under these conditions, $V_{L}$ takes values very close to those obtained for $V_{C}$ when biasing $V_{L}=-V_{R}=V<0$, due to the similar penetration of carriers into the measuring branch. The same explanation can be given for the behavior of $V_{R}$ just changing the sign of $V$. In contrast with this case, for $\alpha=120^{\circ}$, the symmetry is recovered since the orientation of the branches does not originate differences of electron injection into the measuring branch between positive and negative values of $V$.

\section{Ballistic Rectifier}

A new concept for the fabrication of a ballistic rectifying device has been introduced in [2] and [4] by inserting a triangular scatterer (antidot) into the center of a ballistic cross junction. Fig. 9(a) shows the values of the potential between the bottom and top branches $\left(V_{B T}=V_{B}-V_{T}\right)$ when biasing left and right branches in push-pull fashion $V=V_{L}=-V_{R}$ obtained from the MC simulation of the device sketched in the inset. The efficiency of this ballistic rectifier reaches $15 \%$ at $V=0.2 \mathrm{~V}$, in 

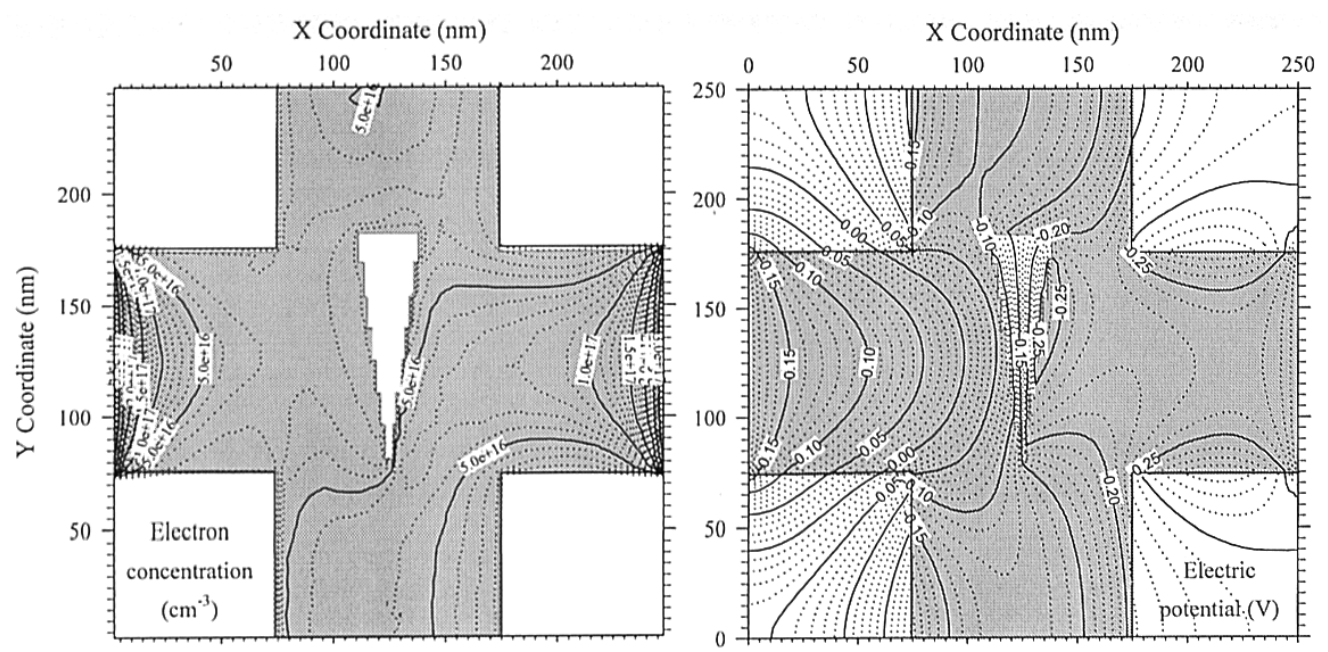

Fig. 10. Two-dimensional contour plots of (a) electron concentration and (b) electric potential in the ballistic rectifier of Fig. 9 for $V=V_{L}=-V_{R}=0.2 \mathrm{~V}$.

good agreement with the experimental results shown in [4]. As shown before for the YBJs, when biasing right and left branches, a negative potential appears in the central branch. In this case, it is generated in both central branches (bottom and top) but with different values due to the unequal width of the opening space between the horizontal and the top/bottom branches [Figs. 9(b) and (c), respectively], leading to an asymmetric injection of carriers into them. The narrower the space between the horizontal and the vertical branch, the higher the potential barrier created by the lateral surface charge, and the lower the probability of an electron to pass from one branch to the other, or following the formalism of [9], the transmission coefficient between those probes is smaller. This can be clearly observed in the 2-D charts of electron concentration and potential in the device shown in Fig. 10. While the $V_{T}$ values are similar to those previously shown for TBJs [Fig. 3(a)] due to the small carrier penetration into the top branch, the stronger injection of carriers into the bottom branch (because of the asymmetric geometry of the obstacle) enhances the curvature of the $V_{B}$ values (like in a YBJ). Therefore, since the curvature of $V_{B}$ is higher than that of $V_{T}$, negative values for $V_{B T}$ are obtained, which depend mainly on the size and shape of both the obstacle and the different branches. Remarkably, even if the values of both $V_{B}$ and $V_{T}$ depend on the surface charge, their difference $V_{B T}$ is almost constant, as observed in Fig. 9. This happens because the different curvature of $V_{B}$ with respect to $V_{T}$ comes from the unequal penetration of carriers into the top and bottom branches, and this difference remains nearly unchanged with $\sigma$ in the range of $\sigma$ values reported in the figure. As in the case of TBJs and YBJs, the behavior of $V_{B}$ and $V_{T}$ (and thus of $V_{B T}$ ) for low $V$ remains parabolic up to a given biasing, for which the unbalanced injection of charge into the top and bottom branches reaches a saturation regime and $V_{B T}$ takes a constant value.

The origin of the rectifying behavior of this device can be therefore understood as a consequence of i) the horizontal asymmetry of the electron concentration associated with both the ballistic transport and space charge inside the device (as in the case of the TBJs and YBJs) and ii) the different openings between the horizontal and top/bottom branches (or, following the formalism of [9], the different transmission coefficients between

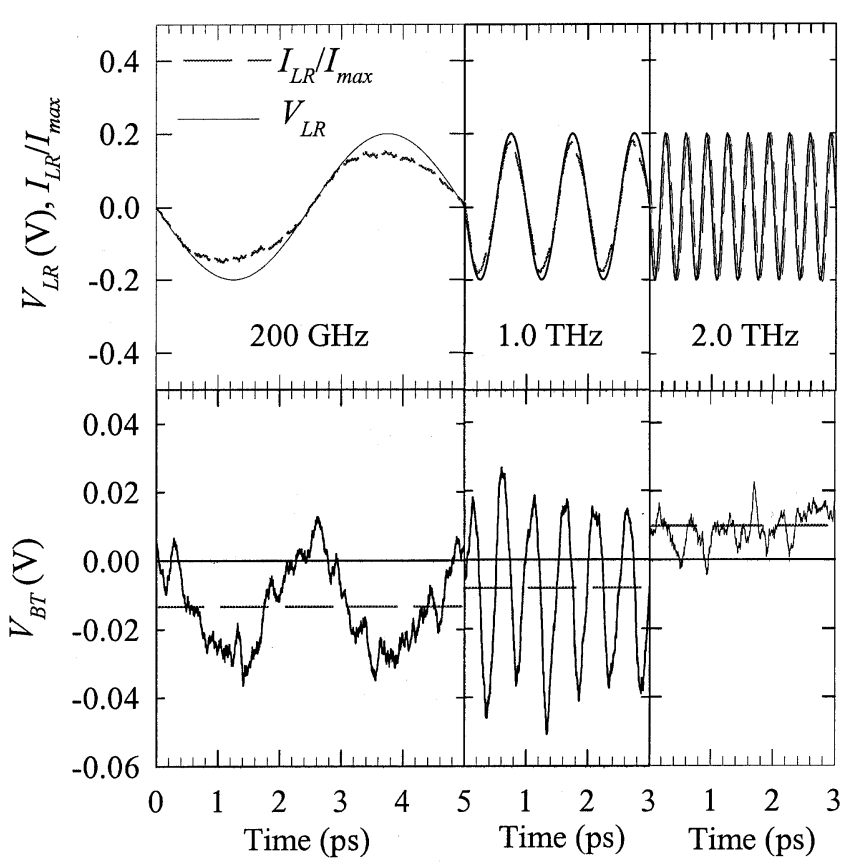

Fig. 11. $V_{B T}$ response to periodic signals with amplitude of $0.2 \mathrm{~V}$ and frequencies of $200 \mathrm{GHz}, 1 \mathrm{THz}$, and $2 \mathrm{THz}$ applied to $V_{L R}$ (potential difference between left ad right electrodes) in the ballistic rectifier of Fig. 9.

them). These two factors originate from the observed vertical asymmetry of electron concentration and, thus, of electric potential.

In order to demonstrate the intrinsic capability of this device for rectification at extremely high frequencies, the $V_{B T}$ response to periodic ac signals with amplitude of $0.2 \mathrm{~V}$ and frequencies of $200 \mathrm{GHz}, 1.0 \mathrm{THz}$, and $2.0 \mathrm{THz}$ applied between left and right electrodes are plotted in Fig. 11. The excellent performance as frequency doubler or power detector in the terahertz range (at least up to $1 \mathrm{THz}$ ) is illustrated in the figure. Moreover, by reducing the size and optimizing the geometry of the device, its intrinsic cutoff frequency, sensitivity, and linearity could be further improved. For this sake, our MC simulator appears as an extremely useful tool since any kind of geometry can 
be easily implemented with no need of a technological process, thus avoiding a great waste of time and money.

\section{CONCLUSIONS}

By using a Monte Carlo simulator, a semiclassical analysis of ballistic transport in a variety of basic mesoscopic devices (AlInAs/InGaAs-based ballistic TBJs, YBJs, and rectifiers) has been performed. The experimental measurements of nonlinear ballistic effects reported in the literature (such as the appearance of a negative potential in the central branch of TBJs and YBJs when biasing $V=V_{L}=-V_{R}$ ) have been qualitatively reproduced and explained, taking as a base the presence of space charge inside the devices, which is strongly influenced by the effect that the surface charge exerts on the electron concentration in such small structures. In our simulations, phase coherence is not necessary for the appearance of the measured ballistic effects. Thus, a semiclassical transport description can be enough to analyze the performance of these devices.

MC simulations also allow the study of the dynamic behavior of these ballistic devices, which have exhibited intrinsic cut-off frequencies in the terahertz range. The flexibility of our simulator to model any kind of geometry makes it an excellent tool for the optimization of the device performance with no need for a (long and expensive) technological process.

\section{REFERENCES}

[1] L. Worschech, H. Q. Xu, A. Forchel, and L. Samuelson, "Bias-voltageinduced asymmetry in nanoelectronic Y-branches," Appl Phys. Lett., vol. 79, pp. 3287-3289, 2001.

[2] A. M. Song, A. Lorke, A. Kriele, J. P. Kothaus, W. Wegscheider, and M. Bichler, "Nonlinear electron transport in an asymmetric microjunction: a ballistic rectifier," Phys. Rev. Lett., vol. 80, pp. 3831-3834, 1998.

[3] K. Hieke and M. Ulfward, "Nonlinear operation of the Y-branch switch: ballistic switching mode at room temperature," Phys. Rev. B, vol. 62, pp. 16727-16730, 2000

[4] A. M. Song, P. Omling, L. Samuelson, W. Seifert, I. Shorubalko, and H. Zirath, "Operation of InGaAs/InP-based ballistic rectifiers at room temperature and frequencies up to $50 \mathrm{GHz}$," Jpn. J. Appl. Phys., vol. 40, pp. L909-L911, 2001.

[5] I. Shorubalko, H. Q. Xu, I. Maximov, D. Nilsson, P. Omling, L. Samuelson, and W. Seifert, "A novel frequency-multiplication device based on three-terminal ballistic junction," IEEE Electron. Device Lett., vol. 23, pp. 377-379, July 2002.

[6] I. Shorubalko, H. Q. Xu, I. Maximov, P. Omling, L. Samuelson, and W. Seifert, "Nonlinear operation of GaInAs/InP-based three-terminal ballistic junctions," Appl. Phys. Lett., vol. 79, pp. 1384-1386, 2001.

[7] Y. Yamashita, A. Endoh, K. Shinohara, K. Hikosaka, T. Matsui, S. Hiyamizu, and T. Mimura, "Pseudomorphic $\mathrm{In}_{0.52} \mathrm{Al}_{0.48} \mathrm{As} / \mathrm{In}_{0.7} \mathrm{Ga}_{0.3}$ As HEMT's with an ultrahigh $f_{t}$ of 562 GHz," IEEE Electron Device Lett., vol. 23, pp. 573-575, Oct. 2002.

[8] J. O. Wesström, "Self-gating effect in the electron Y-branch switch," Phys. Rev. Lett., vol. 82, pp. 2564-2567, 1999.

[9] A. M. Song, "Formalism of nonlinear transport in mesoscopic conductors," Phys. Rev. B, vol. 59, pp. 9806-9809, 1999.

[10] H. Q. Xu, "Electrical properties of three-terminal ballistic junctions," Appl. Phys. Lett., vol. 78, pp. 2064-2066, 2001.

[11] R. Landauer, "Spatial variation of currents and fields due to localized scatterers in metallic conduction," IBM J. Res. Dev., vol. 1, pp. 223-231, 1957.

[12] M. Büttiker, "4 terminal phase coherent conductance," Phys. Rev. Lett., vol. 57, pp. 1761-1764, 1986.

[13] J. Mateos, T. González, D. Pardo, V. Hoel, and A. Cappy, "Improved Monte Carlo algorithm for the simulation of $\delta$-doped AlInAs/GaInAs HEMTs," IEEE Trans. Electron Devices, vol. 47, pp. 250-253, Jan. 2000 .
[14] — "Effect of the T-gate on the performance of recessed HEMT's. A Monte Carlo analysis," Semicond Sci. Technol., vol. 14, pp. 864-870, 1999.

[15] _ - "Monte Carlo simulator for the design optimization of low-noise HEMTs," IEEE Trans. Electron Devices, vol. 47, pp. 1950-1956, Oct. 2000.

[16] T. González, O. M. Bulashenko, J. Mateos, D. Pardo, and L. Reggiani, "Effect of long-range Coulomb interaction on shot-noise suppression in ballistic transport," Phys. Rev. B, vol. 56, pp. 6424-6427, 1997.

[17] S. Reitzenstein, L. Worschech, P. Hartmann, and A. Forchel, "Logic AND/NAND gates based on three-terminal ballistic junctions," Electron. Lett., vol. 38, pp. 951-953, 2002.

[18] R. Lewén, I. Maximov, I. Shorubalko, L. Samuelson, L. Thylén, and H. Q. Xu, "High frequency characterization of a GaInAs/InP electronic waveguide T-branch switch," J. Appl. Phys., vol. 91, pp. 2398-2402, 2002.

[19] H. Q. Xu, "A novel electrical property of three-terminal ballistic junctions and its applications to nanoelectronics," Physica E, vol. 13, pp. 942-945, 2002.

[20] T. González, O. M. Bulashenko, J. Mateos, D. Pardo, and L. Reggiani, "Microscopic analysis of shot-noise suppression in nondegenerate ballistic transport," Semicond. Sci. Technol., vol. 13, pp. 714-724, 1998.

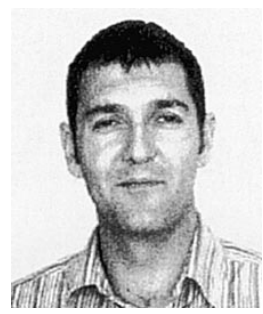

Javier Mateos was born in Salamanca, Spain, in 1970. He received the B.S. and Ph.D. degrees in physics from the University of Salamanca in 1993 and 1997, respectively.

Since 1993, his has been working with the Electronics Group, Department of Applied Physics, University of Salamanca, as a Grant Holder. In 1996, he became Assistant Professor. He worked for one year at the Institut d'Electronique, de Microélectronique et de Nanotechnologies (IEMN), Villeneuve D'Ascq, France. In 2000, he became Associate Professor. His present research interest is in the development of novel device concepts using ballistic transport, together with the modeling and optimization of the high-frequency and low-noise performance of ultra-short gate-length HEMTs

B. G. Vasallo, photograph and biography not available at time of publication.

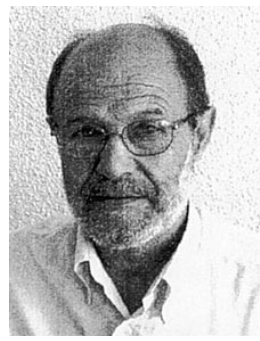

Daniel Pardo was born in Valladolid, Spain, in 1946. He graduated in physics in 1971 from the University of Valladolid, where he also received the Ph.D. degree in 1975.

From 1971 to 1981, he was working in the Electronics Department of the University of Valladolid on the characterization of semiconductor materials and modeling of semiconductor devices, where he was promoted to Associate Professor in 1978. In 1981, he joined the Applied Physics Department, University of Salamanca, Salmanca, Spain, where he has been Full Professor and Head of the Electronics Group since 1983. His current research interest is the Monte Carlo simulation of semiconductor devices with special application to noise characterization.

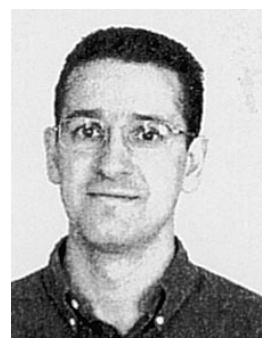

Tomás González was born in Salamanca, Spain, in 1967. He graduated in physics from the University of Salamanca in 1990, where he received the Ph.D. degree in physics in 1994

Since 1991, he has been with the Electronics Group, Department of Applied Physics, University of Salamanca, with a grant from the Spanish Education Ministry. In 1996, he became Associate Professor. His main research activity is in the field of electronic transport in semiconductor materials, electronic devices, and mesoscopic structures, with special application to the modeling of electronic noise by microscopic approaches. 


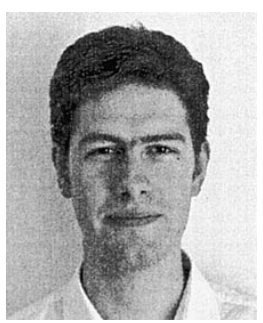

Jean-Sébastien Galloo was born in Seclin, France, on October 13, 1978. He studied physics at the University of Lens, Lens, France, then Electronics in 2001 at the University of Lille, Lille, France. In 2002, he began to pursue the Ph.D. degree at the Institut d'Electronique, de Microélectronique et de Nanotechnologies (IEMN), Villeneuve d'Ascq, France. His doctoral dissertation is entitled "Ballistic Nanodevices for Terahertz Data Processing."

He is involved in simulation, realization, and characterization of ballistic devices.

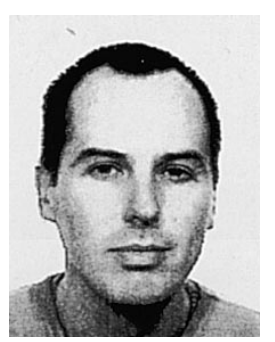

Sylvain Bollaert was born in Calais, France, on February 17,1965 . He received the Ph.D. degree from the University of Lille, Lille, France, in 1994.

$\mathrm{He}$ is an Associate Professor with the Institut d'Electronique, de Microélectronique et de Nanotechnologies (IEMN), Villeneuve d'Ascq, France. His main research interest is the fabrication of nanoscaled devices and monolithic microwave integrated circuits. For the last three years, he has developed the fabrication process for 50 -nm gate length HEMTs using InAlAs/InGaAs lattice-matched and pseudomorphic on InP and metamorphic on GaAs. He is currently involved in the realization of ultra-high speed MMICs using such devices and in the development of sub-50-nm gate length HEMTs. He has also worked on the study and the realization of ballistic devices: transferred-substrate HEMTs for terahertz frequency applications.

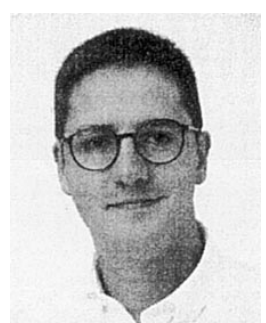

Yannick Roelens was born in Villeneuve d'Ascq, France, on May 3, 1972. He received the Ph.D. degree from the University of Lille, Lille, France, in 2000. His Ph.D. dissertation was on application of High-Tc superconductors in microwaves.

Since September 2001, he has been an Associate Professor with the Institut d'Electronique, de Microélectronique et de Nanotechnologies (IEMN), Villeneuve d'Ascq. His main research interest is the fabrication of nanoscaled devices and microwave circuits. $\mathrm{He}$ is involved in the realization and characterization of ballistic devices.

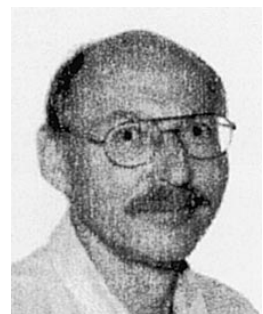

Alain Cappy was born in Chalons sur Mame, France, on January 25, 1954. In 1977, he joined the Institut d'Electronique, de Microélectronique et de Nanotechnologies (IEMN), University of Lille, Villeneuve d'Ascq, France, where he received the Dr.Sci. degree for his work on the modeling and the characterization of MESFETs and HEMTs in 1986.

He is presently Director of the IEMN and Professor of electronics and electrical engineering at the University of Lille. His main research interests are concerned with the modeling, realization, and characterization of ultra-high-speed devices and circuits for applications in the centimeter and millimeter-wave ranges. 